(2) 


\title{
ANNALEN \\ DER DEUTSCHEN \\ LITERATUR
}

\author{
GESCHICHTE DER DEUTSCHEN \\ LITERATUR \\ VON DEN ANFÄNGEN BIS ZUR GEGENWART \\ EINE \\ GEMEINSCHAFTSARBEIT ZAHLREICHER \\ FACHGELEHRTER \\ HERAUSGEGEBEN VON \\ HEINZ OTTO BURGER
}

MCMLII

J. B. METZLERSCHE VERLAGSBUCHHANDLUNG

STUTTGART 
ISBN 978-3-476-99182-9

ISBN 978-3-476-99181-2 (eBook)

DOI 10.1007/978-3-476-99181-2

C 1952 Springer-Verlag GmbH Deutschland

Ursprünglich erschienen bei J. B. Metzlersche Verlagsbuchhandlung und Carl Ernst Poeschel Verlag GmbH, Stuttgart. 
$J$ ede Zeit versucht stets aufs neue, ihr eigenes Bild von der Geschichte, auch der Literaturgeschichte, zu gewinnen. Nach dem inneren und äußeren Zusammenbruch der letzten Jahre ist dieser Wunsch besonders dringlich geworden. Die Literaturwissenschaft aber hat sich inzwischen, schon seit vielen Jahrzehnten, so spezialisiert, daß eine wirklich auf dem heutigen Stand der Forschung aufgebaute Gesamtdarstellung der Geschichte der deutschen Literatur von einem Einzelnen kaum mehr geleistet werden kann. Darum ist das vorliegende Buch die Gemeinschaftsarbeit eines größeren Kreises von Fachgelehrten.

Ein Sammelwerk, das die Freiheit der Mitarbeiter respektiert - in unserem Falle eine Selbstverständlichkeit -, setzt sich immer der Gefahr aus, Bilder aneinanderzureihen, die unter völlig verschiedener Perspektive gesehen sind. In der annalistischen Ordnung findet das vorliegende Buch ein zugleich lockeres und festes Band, das, wie nicht leicht ein anderes, Einheit in der Vielheit und Vielheit in der Einheit ermöglicht.

Die Verfasser der 'Annalen der deutschen Literatur' haben als Hochschullehrer vor allem an die Bedürfnisse der Studenten gedacht; doch trifft ja, was der Student "braucht", weithin zusammen mit dem, was auch der Deutschlehrer der Höheren Schule, der Bibliothekar, der Buchhändler, der Liebhaber suchen. Und weil an den deutschen Universitäten Lehre und Forschung zusammengehören, so hoffen wir, mit den 'Annalen' nicht zuletzt die Wissenschaft selbst in manchen Punkten gefördert zu haben.

Das $A$ und $O$ der Literaturwissenschaft heißt heute Interpretation. Um nicht am Einfall, am Zufall hängenzubleiben, braucht die Interpretation ein übergreifendes Sinngefüge, aber dieses wird in der Regel in irgendeiner Typologie, Anthropologie, Ontologie gesucht. Obwohl man sie philosophisch untermauert, hat doch keine von ihnen eine verbindliche Kraft. Die bunte Vielfalt isoliert und relativiert vielmehr die Leistung des einzelnen Forschers. Der Literaturwissenschaft droht von daher die Gefahr, sich in lauter verschiedene, mitunter äußerst geistreiche Versuche, in Essays aufzulösen. Mit der, wenngleich erschütterten, Autorität der Geschichte kann keine der Typologien, Anthropologien oder Ontologien sich messen. Nur der Bezug auf die Geschichte, die Arbeit am Geschichtsbild vermag eine gewisse Einheit der Literaturwissenschaft zu gewährleisten. 
So wichtig dieser Gesichtspunkt ist, er allein brauchte nicht ausschlaggebend zu sein, wenn es nicht eben zum Wesen der Literatur samt der Dichtung gehörte, daß sie in hohem Maße aus den Bedingungen ihrer Zeit erwächst und mit dem Wandel der Zeit sogar ihre Funktionen wechselt. Die Literatur steht nun einmal unter dem Gesetz der Geschichte. Ohne das zu berücksichtigen, kann man sie nicht „verstehen". Was immer die Literaturwissenschaft sonst noch sei, sie muß jedenfalls immer auch Geschichtswissenschaft bleiben.

Andererseits darf sie freilich nicht die Historie zum Selbstzweck machen, so daß die einzelne Dichtung nur mehr als Dokument zählt, statt als Kunstwerk zu gelten. Gegenstand der Literaturwissenschaft ist die Dichtung als ein mit seinem Dasein zu allen Zeiten auf den Menschen wirkendes Werk; gerade um sie als solches zu erschließen und zu begreifen, mu $\beta$ die Literaturwissenschaft in der Dichtung zugleich das geschichtlich gewirkte Werk erkennen. Die Literaturwissenschaft drängt deshalb nach der Synthese von, sagen wir: phänomenologischer Interpretation und Historie, in der Weise, daß die Interpretation des Kunstwerks in seinem überzeitlichen Dasein die Tatsache geschichtlichen Gewordenseins mit einbegreift und die Historie sich ausrichtet an den Kunstwerken, wie sie da sind, gelten und wirken. Das Ideal ist demnach auf neuer Stufe die Einheit von Interpretation und Historie.

Die methodischen Probleme der Interpretation können hier nicht untersucht werden. Jedenfalls fordert die Interpretation größeren Abstand der Literaturwissenschaft von der reinen Geschichtswissenschaft. Aber ist denn die Literaturwissenschaft als Literaturgeschichte jemals so "reine" Geschichtswissenschaft gewesen? Ebenso wie für unsere interpretatorische bedürfen wir auch für unsere historische Aufgabe immer wieder der methodischen Besinnung. Die Literaturgeschichte, wie wir sie betreiben, könnte nicht nur zuviel Geschichte, vom Standpunkt der Interpretation aus, sein, sondern auch zu wenig Geschichte. Der große Althistoriker Eduard Meyer hat immer wieder betont : „Das Auge des Historikers ist die Chronologie." Die Literaturgeschichte zeigt Jahreszahlen gegenüber meist eine starke Aversion, um nicht zu sagen Idiosynkrasie, und springt infolgedessen mit der Chronologie recht großzügig um. Sie geht auf einzelne Dichtergestalten oder auf große Strömungen, Richtungen, Räume aus, und für diese sinnhaften Strukturen bedeuten ihr die Zäsuren der "mechanischen" Zeit wenig. Tatsächlich ist aber in der Regel für den Literaturhistoriker nächst dem Werk dessen Erscheinungsjahr die erste und sicherste Gegebenheit. Er berücksichtigt sie, das versteht sich von selbst, aber er nimmt sie, wie mir scheint, nicht ernst genug. Die Strukturen, die er von irgendwoher hat oder selbst findet, lösen sich sofort von der scheinbar mechanischen Zeit und setzen eigene Zeitmaße. Die gleiche Jahreszahl, etwa 1857 für Stifters 'Nachsommer' und Flauberts 'Madame Bovary', Hebbels 'Gedichte' und 'Les Fleurs du Mal' von Baudelaire, wird, sofern überhaupt gesehen, wohl immer als "Zufall" behandelt. Wie wäre es, wenn man die "mechanische" Gleichzeitigkeit einmal wirklich ernst nähme, mindestens als heuristisches Prinzip?

Noch nie hat meines Wissens die Literaturwissenschaft von unten angefangen, das heißt dort, wo die Geschichtsschreibung herkommt und wohin die Geschichtswissenschaft immer wieder einkehrt: bei der einfachen Annalistik. Da die Literaturgeschichte 
das "Auge" der Chronologie so wenig in Gebrauch nahm, fehlt ihr, jedenfalls was die Studenten unserer Wissenschaft anlangt, die scharfe und genaue Sicht sowohl des historischen Nacheinanders als namentlich des historischen Nebeneinanders. Das vorliegende Buch möchte deshalb Chronologie und Synchronismus der Literaturwerke einmal besonders herausarbeiten. Den Vorwurf des "Neu-Positivismus" fürchten wir nicht.

Auch uns geht es ja um sinnhafte Strukturen. Nur suchen wir diese nicht so sehr im Wurzelgeflecht, aus dem die Werke hervorgewachsen sind, als vor allem im Baumstand der Werke selbst. Es gilt, gleichsam die Zeittafel der Werke zu lesen, und zwar möglichst unvoreingenommen, das hei $\beta$ t so, da $\beta$ Interpretation der Werke und Interpretation der Zeittafel Hand in Hand gehen. Die Werke treten bei diesem chronologischen und synchronistischen Aspekt nicht selten in ganz neue Konstellationen. Die Jahreszahlen, die Zäsuren der „mechanischen"Zeit, ergeben selbst eine Strukturierung und Periodisierung. Die mechanische Zeit offenbart sich als schicksalhafte, geschichtliche Zeit.

Eine Periodisierung, die auf solche induktive Weise gewonnen wird, ist kleinteiliger und insofern genauer als die übliche. Sie stürzt diese nicht um, aber sie wandelt sie da und dort ab. Vielleicht verliert sich stellenweise die durchgehende Linie; da für werden an anderen Stellen, im Kleinen wie im Großen, auf einmal überraschende Bilder und Zusammenhänge sichtbar. Daß die Gestalten der Dichter ihre plastische Geschlossenheit bis zu einem gewissen Grade aufgeben müssen, indem sie in das Gesamtrelief der Dichtungsgeschichte als Werkgeschichte zurücktreten, gehörtzumWesender Methode. Nur wo bekannte Durchblicke verdeckt werden, öffnet sich ein neuer.

Der Plan zu den 'Annalen der deutschen Literatur' stammt vom Herausgeber. Für die Verwirklichung bat er einen Kreis von zuständigen Kollegen um ihre Mitarbeit. Jeder hat sich nach seiner Art und entsprechend der Forschungslage auf seinem Gebiet mit dem annalistischen Prinzip selbständig auseinandergesetzt.

Da der ursprüngliche Plan, die Geschichte der deutschen Dichtung im Zusammenhang der gesamten abendländischen Kultur zu zeigen, sich schon aus Raummangel nur gelegentlich durchführen ließ, wird dem Buch eine eingehende Zeittafel zum Selbst-,,Lesen" als Ergänzung beigegeben. Auch die Bibliographie erscheint in einem besonderen Band.

Den letzten Abschnitt des Textes hatte Werner Milch übernommen; der Tod entri $\beta$ ihn uns, ehe er etwas zu Papier bringen konnte. Für die Zeit von 1230 bis 1490 war Friedrich Ranke gewonnen worden. Wie für alle, die ihn persönlich kannten, und für die gesamte Wissenschaft bedeutete sein Tod auch für die 'Annalen' einen schweren Schlag. Das Letzte, was Friedrich Ranke geschrieben hat, ist sein Beitrag zu diesen 'Annalen', der jedoch mit dem Jahre 1430 abbricht. Infolgedessen mußte für die Jahre 1430 bis 1490 ein besonderes Kapitel eingeschoben werden.

Dem Andenken Friedrich Rankes sei das Buch gewidmet! 
VORGESCHICHTLICHE UND FRÜHGESCHICHTLICHE ZEIT

2000- 770. Von Prof. Dr. Felix Genzmer, Universität Tübingen . . . . . . . . 1

I. $2000-500$ : Urzeit $\ldots \ldots \ldots \ldots \ldots \ldots \ldots \ldots \ldots \ldots \ldots \ldots \ldots \ldots$

II. $\quad 500-200$ : Entstehung eines besonderen germanischen Stils $\ldots \ldots \ldots \ldots$

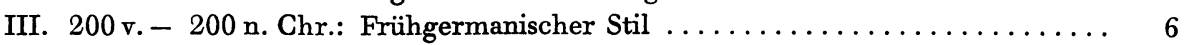

IV. $\quad 200-450:$ Germanisch-frühklassische Zeit $\ldots \ldots \ldots \ldots \ldots \ldots \ldots \ldots \ldots$

V. $\quad 450-600$ : Germanisch-hochklassische Zeit $\ldots \ldots \ldots \ldots \ldots \ldots \ldots \ldots \ldots$

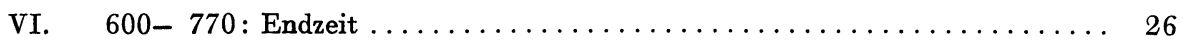

VON DER KAROLINGISCHEN ZUR CLUNIAZENSISCHEN EPOCHE 770-1170. Von Prof. Dr. Helmut de Boor, Freie Universität Berlin . . . . . . 31

I. $770-910$ : Die karolingische Literatur $\ldots \ldots \ldots \ldots \ldots \ldots \ldots \ldots \ldots \ldots \ldots \ldots$

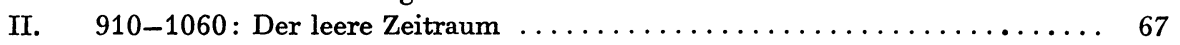

III. 1060-1170: Die cluniazensisch-frühmittelhochdeutsche Literatur . . . . . . 69

DIE KLASSIK DES RITTERTUMS IN DER STAUFERZEIT 1170-1230. Von Prof. Dr. Hugo Kuhn, Universität Tübingen . . . . . . . . . 99

I. 1170-1180: Der symbolische Naturalismus frühhöfischer Dichtung. . . . . . . 102

II. 1180-1190: Weltdienst und Gottesdienst als Analogie und Gegensatz . . . . 113

III. 1190-1200: Die Kunstwelt des höfischen Idealismus . . . . . . . . . . . . 127

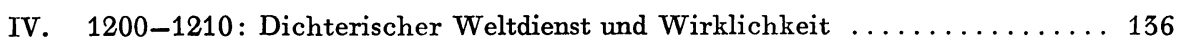

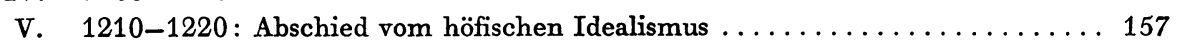

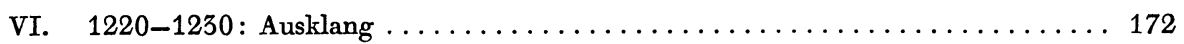

VON DER RITTERLICHEN ZUR BÜRGERLICHEN DICHTUNG 1230-1430. Von Prof. Dr. Friedrich Ranke, Universität Basel . . . . . . . . . 179

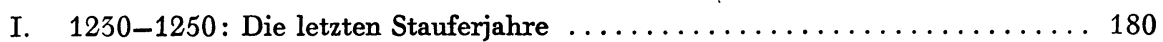

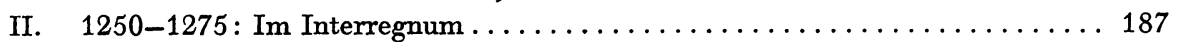

III. 1275-1300: Unter Rudolf von Habsburg und Adolf von Nassau . . . . . . . . 194

IV. 1300-1325: Zwiespalt in Staat und Kirche; Einssein mit Gott . . . . . . . . 205

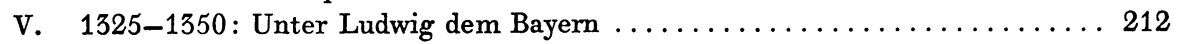

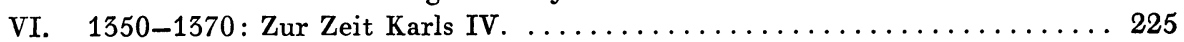

VII. 1370-1400: Um Gottesfreund vom Oberland und Mönch von Salzburg . .. 233

VIII. 1400-1410: Ein Jahrzehnt der großen Dichtungen . . . . . . . . . . 241

IX. 1410-1430: Konstanzer Konzil und Hussitenkriege . . . . . . . . . . 247 
STÄDTE, HÖFE, GELEHRTE

1430-1490. Von Prof. Dr. Siegfried Beyschlag, Universität Erlangen ....... 255

I. 1430-1450: Die Grundlage; Spiel und Literatur der städtischen Gemein-

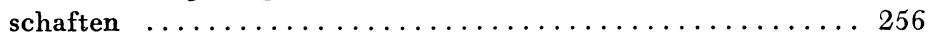

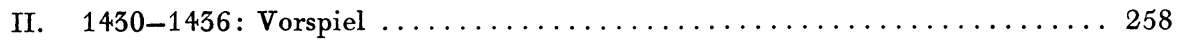

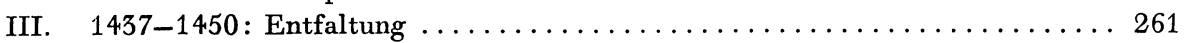

IV. 1450-1460: Scheitelhöhe des Jahrhunderts; Neue Richtungen ........ 265

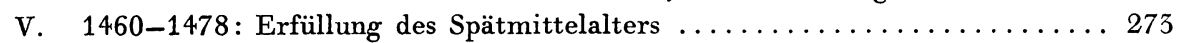

VI. 1479-1490: Ausklang und neue Ansätze $\ldots \ldots \ldots \ldots \ldots \ldots \ldots \ldots \ldots \ldots$

\section{HUMANISMUS UND REFORMATION}

1490-1600. Von Prof. Dr. Richard Newald, Universität Freiburg i. Br. . . . . 287

I. 1490-1500: Alemannische Narrendichtung; Humanistische Anregungen . . . 289

II. 1500-1509: Neue Formen, Streit und theoretische Grundlegungen ...... 294

III. 1510-1520: Das Jahrzehnt der Entscheidungen ............... 296

IV. 1520-1531: Das Schrifttum im Kampf um die Reformation .......... 306

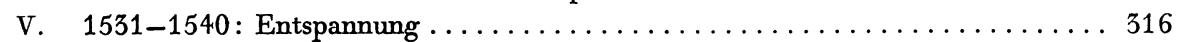

VI. 1540-1550: Gefestigte literarische Überlieferungen ............ 322

VII. 1550-1559: Roman und Schwank ...................... 325

VIII. 1560-1569: Aufflackernde Polemik im Zeichen der Gegenreformation ... 328

IX. 1570-1581: Um Fischart und Frischlin ................... 332

X. 1582-1599: Ausklang ................................... 335

\section{DAS JAHRHUNDERT DES BAROCK}

1600-1700. Von Prof. Dr. Willi Flemming, Universität Mainz ........... 339

I. 1600-1630: Vorläufer und Wegbereiter .................... 341

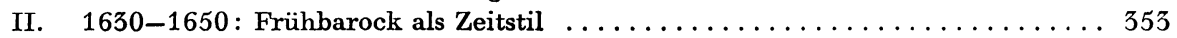

III. 1650-1660: Das erste Jahrzehnt des Hochbarock ................ 362

IV. 1660-1670: Das zweite Jahrzehnt des Hochbarock . . . . . . . . . . . . 377

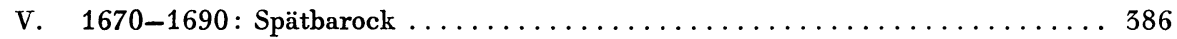

VI. 1690-1700: Das Ende ............................... 401

VON DER AUFKLÄRUNG ZUM STURM UND DRANG

1700-1775. Von Prof. Dr. Fritz Martini, Technische Hochschule Stuttgart . . . . 405

I. 1700-1712: Übergangszeit; Pietismus und Klassizismus, Rationalismus und

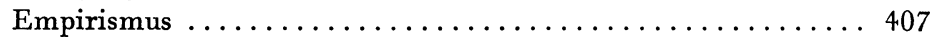

II. 1712-1740: Die Blütezeit der Aufklärung .................... 411

III. 1740-1747 : Das empfindsame Rokoko als Zeitstil ............... 422

IV. 1748-1768: Die Stiftung neuer Maße ....................... 429

V. 1769-1775: Von Herders Abfahrt aus Riga bis zu Goethes Ankunft in Weimar; Sturm und Drang ......................... 447

DIE ZEIT DER KLASSIK UND FRÜHEN ROMANTIK 1775-1805. Von Prof. Dr. Wolfdietrich Rasch, Universität Würzburg ....... 465

I. 1776-1780: Die Peripetie des Sturm und Drang .............. 471

II. 1781-1790: Der Weg zur Klassik ....................... 484 
III. 1791-1797: Polarität und Steigerung .................... 503

IV. 1798-1805: Klassisch-romantische Mannigfaltigkeit $\ldots \ldots \ldots \ldots \ldots \ldots . \ldots 24$

\section{DIE ZEIT DES ALTEN GOETHE}

1805-1832. Von Prof. Dr. Wolfgang Baumgart, Universität Erlangen ...... 551

I. $1805-1808:$ Zeithöhe ............................ 552

II. 1808-1814: Zeitbewußte Romantik ..................... 566

III. $1815-1820$ : Romantische Steigerungen .................. 585

IV. 1821-1826: Gegenromantisches Zeitbewußtsein und romantische Abwehr ... 599

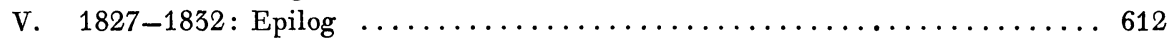

DER REALISMUS DES NEUNZEHNTEN JAHRHUNDERTS

1832-1889. Von Prof. Dr. Heinz Otto Burger, Universität Erlangen ........ 621

I. 1832-1836: Nach Goethes Tod - Die Zeit Büchners ............... 622

II. 1837-1842: Nach Büchners Tod - Reifejahre Stifters ............ 637

III. 1843-1850: Neuromantik und Revolution - Reifejahre Hebbels . . . . . . . 651

IV. 1851-1861: Die Höhe des Jahrhunderts - Reifejahre Kellers . . . . . . . . 666

V. 1862-1871: Nach Uhlands Tod - Reifejahre Raabes .............. 690

VI. 1872-1889: Die Zeit Nietzsches - Reifejahre Fontanes ............ 700

DER WEG INS ZWANZIGSTE JAHRHUNDERT

1889-1945. Von Dr. Hans Schwerte, Universität Erlangen . . . . . . . . . 719

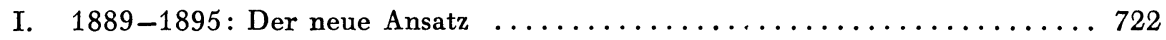

II. 1895-1905: Die Auffächerung des Neuansatzes und Ernte der Jahrhundert-

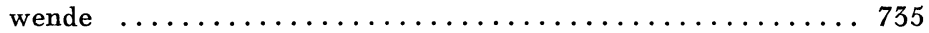

III. 1905-1911: Verfestigung und Auflösung des Ansatzes von 1889 . . . . . . 760

IV. 1911-1924: Der Zeitraum des Expressionismus ................. 785

V. 1924-1945: Desillusionierter Realismus; Neues Sprachexperiment; Fortführung der Altkräfte $\ldots \ldots \ldots \ldots \ldots \ldots \ldots \ldots \ldots \ldots \ldots \ldots \ldots$

\section{ANNALEN DER DEUTSCHEN SPRACHE}

Von Prof. Dr. Hugo Moser, Universität Tübingen .................. 841

I. Bis zur Mitte des 8. Jahrhunderts n. Chr.: Vorgeschichte der deutschen Sprache 841

II. Mitte des 8. bis Anfang des 16. Jahrhunderts : Altdeutsche Zeit . . . . . . . 843

III. Seit dem Anfang des 16. Jahrhunders: Neudeutsche Zeit ........... 849

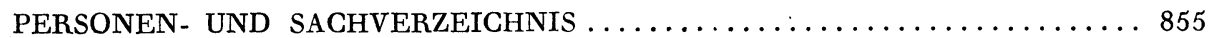

ERGÄNZUNGSHEFT I : VERGLEICHENDE ZEITTAFELN VON DER FRÜHGESCHICHTE BIS ZUR GEGENWART

Von Prof. Dr. Kurt Herbert Halbach, Universität Tübingen ... (erscheint gesondert)

\section{ERGÄNZUNGSHEFT II : BIBLIOGRAPHIE DES GESAMTZEITRAUMES DER} DEUTSCHEN LITERATUR

Von Dr. Otto Olzien, Universitätsbibliothek Göttingen ....... (erscheint gesondert) 